Please do not remove this page

RMIT

UNIVERSITY

\title{
Dynamically reconfigurable plasmon resonances enabled by capillary oscillations of liquid-metal nanodroplets
}

Maksymov, Ivan; Greentree, Andrew

https://researchrepository.rmit.edu.au/esploro/outputs/9921863026901341/filesAndLinks?institution=61RMIT_INST\&index=null

Maksymov, I., \& Greentree, A. (2017). Dynamically reconfigurable plasmon resonances enabled by capillary oscillations of liquid-metal nanodroplets. Physical Review A, 96(4), 1-6.

https://doi.org/10.1103/PhysRevA.96.043829

Document Version: Published Version

Published Version: https://doi.org/10.1103/PhysRevA.96.043829

Repository homepage: https://researchrepository.rmit.edu.au

(C) 2017 American Physical Society

Downloaded On 2023/04/26 13:09:03 +1000

Please do not remove this page 
Thank you for downloading this document from the RMIT Research Repository.

The RMIT Research Repository is an open access database showcasing the research outputs of RMIT University researchers.

RMIT Research Repository: http://researchbank.rmit.edu.au/

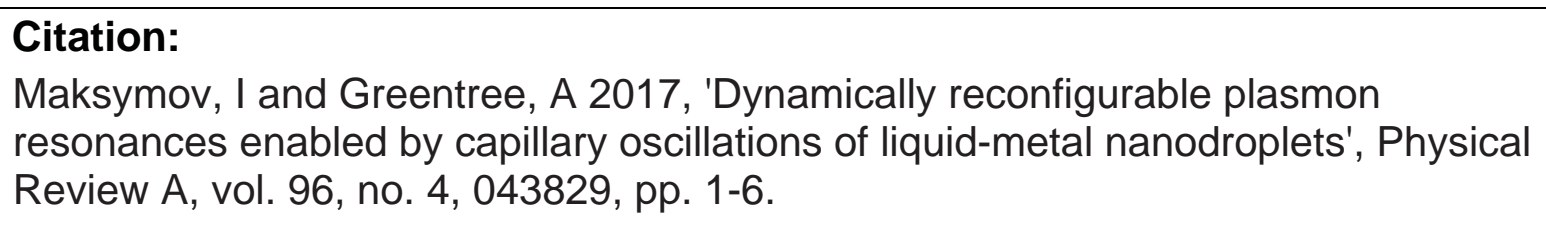

See this record in the RMIT Research Repository at:

https://researchbank.rmit.edu.au/view/rmit:45930

Version: Published Version

\section{Copyright Statement:}

(C) 2017 American Physical Society

Link to Published Version:

https://dx.doi.org/10.1103/PhysRevA.96.043829 


\title{
Dynamically reconfigurable plasmon resonances enabled by capillary oscillations of liquid-metal nanodroplets
}

\author{
Ivan S. Maksymov and Andrew D. Greentree \\ Australian Research Council Centre of Excellence for Nanoscale BioPhotonics, School of Science, RMIT University, Melbourne, Victoria \\ 3001, Australia \\ (Received 18 July 2017; revised manuscript received 19 September 2017; published 12 October 2017)
}

\begin{abstract}
Plasmonics allows manipulating light at the nanoscale, but has limitations due to the static nature of nanostructures and lack of reconfigurability. We propose and theoretically analyze a room-temperature liquidmetal nanodroplet that changes its shape, and therefore dynamically reconfigures the plasmon resonance spectrum, due to capillary oscillations. We show the possibility to control the capillary oscillation frequency of the nanodroplet and to drive the oscillations electrically or mechanically. Employed as a dynamically reconfigurable nanoantenna, the nanodroplet may find applications in sensors, imaging, microscopy, and medicine.
\end{abstract}

DOI: 10.1103/PhysRevA.96.043829

\section{INTRODUCTION}

Nanoplasmonics paves the way for controlling light at the subwavelength scale [1]. However, once a metal nanostructure has been fabricated, its optical characteristics cannot be changed in a reversible manner, which restricts its potential applications and does not allow meeting an increasing demand for tunable, and more broadly, dynamically reconfigurable optical properties.

Therefore, a large and growing body of research investigates reconfigurable plasmonic structures such as, e.g., optical nanoantennae [2,3]. Dynamic reconfigurability of nanoantennae has been demonstrated by combining a metal nanostructure with functional materials such as liquid crystals, metamaterials, elastomers, semiconductors, phase-changing media, nonlinear, and magnetooptical materials (for a review see, e.g., [3-5]). Because of many options in size, material, and features, nanoantennae have been employed in optical filters, sensors, switches, on-chip photonic links, sources of quantum light, and so on, and found applications in imaging, spectroscopy, microscopy, photovoltaics, and medicine [3-5].

Although the terms "tunable" and "reconfigurable" have sometimes been employed interchangeably or complemented one another [6,7], in general "tunable" means the ability to continuously change the plasmon resonance frequency through an external influence or signal. This definition is often used in the adjacent area of metamaterials [8]. Instead, in the following a broader term "reconfigurable" is employed because it means not only tuning of the resonance frequency, but also implies any kind of dynamic control of characteristics of the device through electrical, mechanical, optical, chemical, or other means. This definition is used in the adjacent and well-established area of reconfigurable antennae [9], which can simultaneously change the resonance frequency, shape and linewidth, far-field radiation pattern, impedance, and polarization. According to this definition, the modulation $[10,11]$ and variation $[12,13]$ of plasmon resonances that are especially important in microfluidics-based plasmonic sensing and fractal plasmonic structures may also be considered as a type of dynamic reconfiguration.

In this paper, we propose and theoretically analyze a roomtemperature liquid-metal nanodroplet that changes its shape, and therefore allows reconfiguring the plasmon resonance, due to capillary oscillations. Capillary oscillations of the nanodroplet arise because of a competition between inertia and surface tension [14], and may be driven electrically or mechanically (see, e.g., [15]). In our study, for simplicity and to highlight important physics, the nanodroplet oscillates in a vacuum or air. However, the fundamental physics remains essentially the same in the scenario of the nanodroplet surrounded by a liquid. As the constituent material of the nanodroplet we consider a room-temperature liquid-gallium metal alloy that, in contrast to liquid mercury, is nontoxic and has a relatively low density [16,17]. We demonstrate that, similar to solid-gallium nanoparticles of $\sim 100$-300-nm diameter [18,19], liquid-gallium nanodroplets of comparable size [17] have plasmon resonance frequencies in the ultraviolet spectral range. We also show that the good mechanical properties of liquid-gallium alloys, such as a large and reversibly changeable surface tension and low viscosity $[16,20]$, enable capillary oscillations with a low damping rate and oscillation frequency controllable in the $\mathrm{MHz}-\mathrm{to}-\mathrm{GHz}$ range. By considering the scenario of capillary oscillations driven electrically or mechanically, we calculate that the reconfiguration of the plasmon resonance in the 150-300-nm spectral range is possible with experimentally achievable peak amplitudes of the applied ac voltage or mechanical pressure.

Thus, liquid-metal nanodroplets may operate as dynamically reconfigurable optical nanoantennae and therefore have the same wide range of applications in photonic devices conceivable with nanoantennae [2-5]. We also envision applications in emergent areas such as sensing of sound at the nanoscale [21,22], unconventional photonics [23,24], and detection of mechanical signatures of cells and bacteria [25]. Finally, although our analysis is mostly focused on nanodroplets oscillating in a vacuum or air, our findings are extendable to the case of liquid-metal nanodroplets operating inin vivo biological environments, thereby opening new opportunities for biology and medicine [26].

\section{CAPILLARY OSCILLATIONS}

Applications of microscopic liquid droplets in photonics were previously discussed in [27-29]. For example, in [29] a water-droplet microresonator fabricated on the edge of an 
optical fiber was demonstrated. However, a large size and low optical refractive index ( $n=1.33$ for water) of liquid droplets prevent them from applications at the nanoscale. Moreover, preference has mostly been given to acoustic-like oscillations (MHz-range frequencies) of microscopic liquid droplets rather than to capillary ones ( $\mathrm{kHz}$ range) because of the focus on applications in high-frequency optomechanics [28].

However, the nanoscale size of liquid-metal droplets in combination with a high surface tension $\sigma$ and relatively low density $\rho$ of liquid-gallium alloys $(\sim 10$ and $\sim 6$ times $\sigma$ and $\rho$ of water, respectively [16]) allows achieving capillary oscillations in the $\mathrm{GHz}$-frequency range. Thus, we consider capillary oscillations of a single, initially spherical liquidgallium alloy nanodroplet and analyze the resonance angular frequency $\omega_{l}$ and peak amplitude $A_{l}$ of the capillary oscillation modes with the mode numbers $l$.

In the linear approximation, $\omega_{l}$ of a nanodroplet oscillating in a vacuum or air is given by [14]

$$
\omega_{l}^{2}=l(l-1)(l+2) \sigma /\left(\rho R^{3}\right)
$$

where $\sigma$ and $\rho$ are the surface tension of the liquid metal and density, respectively. The modes $l=0$ and $l=1$ are zero-frequency modes corresponding to the conservation of volume and translational invariance, respectively. The lowest nonzero frequency (fundamental) mode excited in experimental conditions is $l=2$.

The three-dimensional (3D) shapes of the oscillation modes are given by (see, e.g., [15])

$$
r=R\left[1+\left(A_{l} / R\right) \cos \left(\omega_{l} t\right) P_{l}(\cos \theta)\right],
$$

where the coordinate origin is at the center of the nanodroplet, $A_{l} / R$ is the peak amplitude of the $l$ th mode, $t$ is time, $\theta=0, \ldots, 2 \pi$, and $P_{l}(x)=\sum_{m=0}^{M}(-1)^{m} \frac{(2 l-2 m) !}{2^{l} m !(l-m) !(l-2 m) !} x^{l-2 m}$ where $M=l / 2$ or $M=(l-1) / 2$ whichever is an integer.

Figure 1 shows representative oscillation mode shapes through a half-period of oscillation for the modes $l=2, \ldots, 7$ with the peak oscillation amplitude $A_{l} / R=0.3$. Within the half-period of oscillation the nanodroplet assumes the shapes at $T=0$ and $T=1$ corresponding to the largest deviation from the spherical shape $T=1 / 2$ (here $T_{l}=\omega_{l} t$, the index $l$ is omitted for simplicity). For the fundamental $l=2$ mode the shape of the nanodroplet changes from an oblate to prolate spheroid. For $l=3$ it changes from an inverted pyramid to a pyramid, and so on. For the second half of the period the nanodroplet retraces the shapes assumed in the first half.

In general, Eqs. (1) and (2) are valid for linear capillary oscillations of an inviscid and incompressible liquid droplet with the infinitesimal amplitudes $A_{l} / R \rightarrow 0$ [14]. This linear theory was developed by Rayleigh and later extended to take into account viscosity of the droplet and consider the scenario of a viscous liquid droplet immersed in another viscous liquid [30,31].

Small amplitude $\left(A_{l} / R \approx 0.1, \ldots, 0.4\right)$ oscillations were analyzed in [32] and a decrease in $\omega_{l}$ was predicted for the modes $l=2, \ldots, 4$ as $\tilde{\omega}_{l}=\omega_{l}\left[1-\gamma_{l}\left(A_{l} / R\right)^{2}\right]$. The values of $\gamma_{l}$ can be found in [32].

Large amplitude $\left(A_{l} / R \gtrsim 0.4\right)$ nonlinear oscillations of inviscid droplets were investigated in [33], and viscosity was taken into account in [34]. The numerical method proposed in [34] also works in the case of small amplitude oscillations, and together with earlier numerical results [35,36], it confirms the accuracy of the theory from [32].

Using the corrections for the oscillation mode shapes from [32], we analyze the difference between the predictions of the linear and nonlinear theories. At the peak oscillation amplitudes $A_{l} / R=0.4$ considered in $[32,35,36]$ for the modes $l=2, \ldots, 4$, the variations in the shape of the oscillating droplet follow the predictions of the linear theory within $\sim 10 \%$ accuracy, with the maximum deviation occurring near the equator and the poles of the droplet. The most notable difference is observed when the linear theory predicts the return of the droplet to a perfectly spherical shape, but the

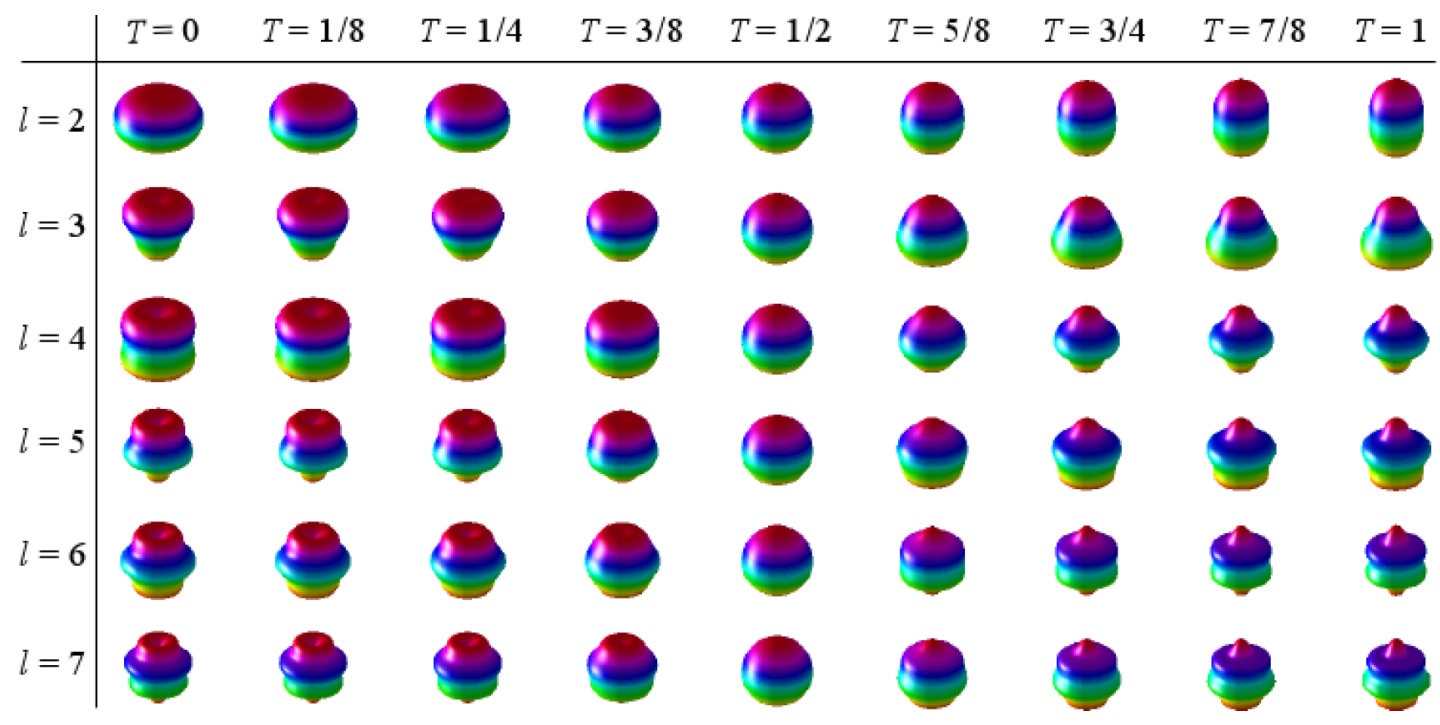

FIG. 1. Representative oscillation mode shapes of plasmonic liquid-gallium alloy nanodroplets through a half-period of oscillation for the $l=2, \ldots, 7$ modes. The peak oscillation amplitude for all modes is $A_{l} / R=0.3$ as justified in the main text. $T_{l}=\omega_{l} t$ is given in the units of $\pi$ radians $\left(T_{l}=1\right.$ is a half of period). 
nonlinear theory produces a prolate spheroid shape for the fundamental mode $(l=2)$ and multilobed shapes for the modes $l=3$ and $l=4$.

Consequently, we expect a $<10 \%$ difference between the oscillation mode shapes in Fig. 1 obtained in the linear approximation and the predictions of the nonlinear theory and simulations in $[32,35,36]$ because the peak amplitude $A_{l} / R=0.3$ used in our analysis is smaller than that in those papers.

Rigorous numerical analysis has to be employed instead of our linear model when quantitative agreement needs to be achieved. This requires software implementation based on a complex Galerkin finite-element technique combined with an implicit predictor-corrector method [34]. However, such software is complex to develop, not readily available, and requires high-performance computational facilities. Therefore, in the following we take advantage of a simple and intuitive linear model because in this work we mostly aim to demonstrate dynamic reconfiguration in the optical domain.

We analyze the shape mode equation $[14,15,30]$ to define the strength of the oscillation driving force $F_{l}$ required to produce the modal shapes in Fig. 1

$$
\ddot{a}_{l}+\frac{2(l-1)(2 l+1) \mu}{\rho R^{2}} \dot{a}_{l}+\frac{l(l-1)(l+2) \sigma}{\rho R^{3}} a_{l}=\frac{l F_{l}}{\rho R},
$$

where $\omega_{l}$ is given by Eq. (1), $\mu$ is the viscosity of the liquid metal, and $a_{l}$ is a function of time $t$ representing the instantaneous amplitude of the $l$ th oscillation mode. For periodic $F_{l}$, Eq. (3) describes a periodically forced damped harmonic oscillator, for which the general solution is the sum of a transient solution and a steady-state response.

We are interested in the amplitude of the steady-state response because it relates the value of $A_{l} / R$ to the peak amplitude of $F_{l}$. As the oscillation force we consider mechanical pressure or applied ac voltage. In the former case, nanodroplets may be surrounded by a liquid and excited by ultrasound. It is noteworthy that the general physics of immersed liquidmetal nanodroplets is essentially no different from that of nanodroplets oscillating in vacuum or air. The scenario of the excitation with ultrasound is schematically illustrated in Fig. 2(a), where an immersible ultrasonic transducer is placed into a water tank and a cuvette with an aqueous solution of liquid-metal nanodroplets is placed in the area of the focal spot of the transducer. The cuvette is made of a material that is transparent for both ultrasound and incident light. (Incident light is shown schematically; see, e.g., [37] for optical characterization of liquid-based samples.) Due to a close similarity between the setup in Fig. 2(a) and experimental equipment employed in photoacoustics, further technical details may be found in works on photoacoustic interactions (see, e.g., [38]). Another scenario of mechanical excitation may be the propulsion of nanodroplets with a catapult [39]. Although this effect has so far been demonstrated for macroscopic droplets only, the behavior of liquid-metal nanodroplets is expected to be similar. Significantly, experimental snapshots of catapulted droplets in [39] demonstrate that they oscillate and accept shapes similar to those in Fig. 1.

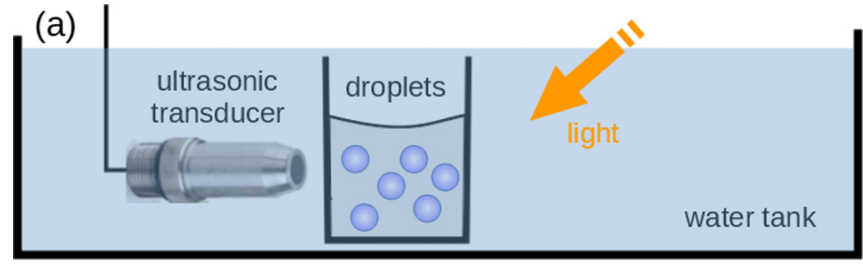

(b)

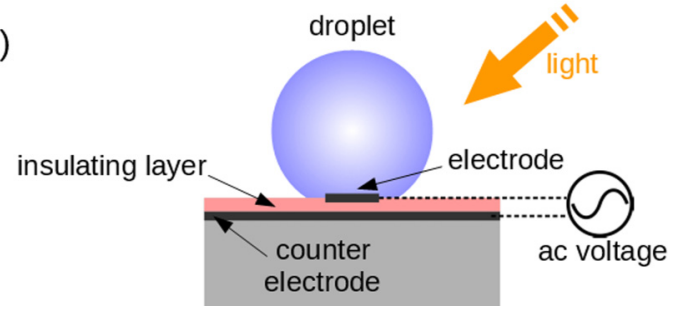

FIG. 2. Schematic illustration of experimental setups for the excitation of capillary oscillation of nanodroplets with (a) ultrasound and (b) ac voltage.

The excitation scenario of applied ac voltage corresponds to electrowetting: the electrical control of wettability used to handle liquid droplets [15], including liquid-metal ones [40,41]. A typical electrowetting setup employs a thin insulating layer (thickness $d$ and radio-frequency range dielectric permittivity $\left.\epsilon_{\mathrm{d}}\right)$ that separates the droplet from the counter electrode. In general, two electrode arrangements can be employed: (i) a fine metal probe and (ii) a planar electrode (see, e.g., [40] for details). In Fig. 2(b), we show a planar configuration because this appears to be more suitable for a nanoscale system as well as minimizes parasitic reflections and plasmon losses from the metal probe.

According to [15], for electrowetting $F_{l}=(2 l+1)$ $\epsilon_{0} \epsilon_{\mathrm{d}} V_{l}^{2} /(2 d R)$, where $\epsilon_{0}$ is the vacuum permittivity and $V_{l}$ is the peak amplitude of the applied ac voltage for the $l$ th mode. However, because in our model the nanodroplet is not attached to a surface, in calculations we use the generic values $d=100 \mathrm{~nm}$ and $\epsilon_{\mathrm{d}}=6$, which may be suitable for the design of real-life devices. At the resonance, for the peak amplitude $A_{l} / R=0.3$ from the steady-state solution to Eq. (3) we obtain $F_{l}=0.3 R^{2} b \omega_{l} \rho / l$ for the mechanical pressure excitation and $V_{l}=\sqrt{0.6 R^{3} b \omega_{l} \rho d /\left[l(2 l+1) \epsilon_{0} \epsilon_{\mathrm{d}}\right]}$ for the ac voltage excitation. Here $b=2(l-1)(2 l+1) \mu /\left(\rho R^{2}\right)$.

\section{RECONFIGURABLE PLASMON RESONANCES}

We use typical liquid-gallium alloy parameters [16,20]: density $\rho=6360 \mathrm{~kg} / \mathrm{m}^{3}$, viscosity $\mu=0.0024 \mathrm{~Pa} \mathrm{~s}$, and surface tension reversibly changeable from $\sigma=0.7 \mathrm{~N} / \mathrm{m}$ down to $0.07 \mathrm{~N} / \mathrm{m}$ (approximately $\sigma$ of water) [20]. The radius of the spherical (undeformed) nanodroplet $R=100$ $\mathrm{nm}$ is chosen because this is a typical size of gallium alloy metal nanodrolets that can be achieved by sonicating the metal in an aqueous solution [17]. Solid-gallium nanoparticles of comparable size support plasmon resonances in the ultraviolet spectral range, in which the dielectric functions of liquid and solid gallium are nearly identical $[19,42]$.

Figure 3(a) shows the capillary resonant oscillation frequency $f_{l}=\frac{\omega_{l}}{2 \pi}$ as a function of the surface tension $\sigma$ for the modes $l=2, \ldots, 7$. Figure 3(b) shows the voltage 

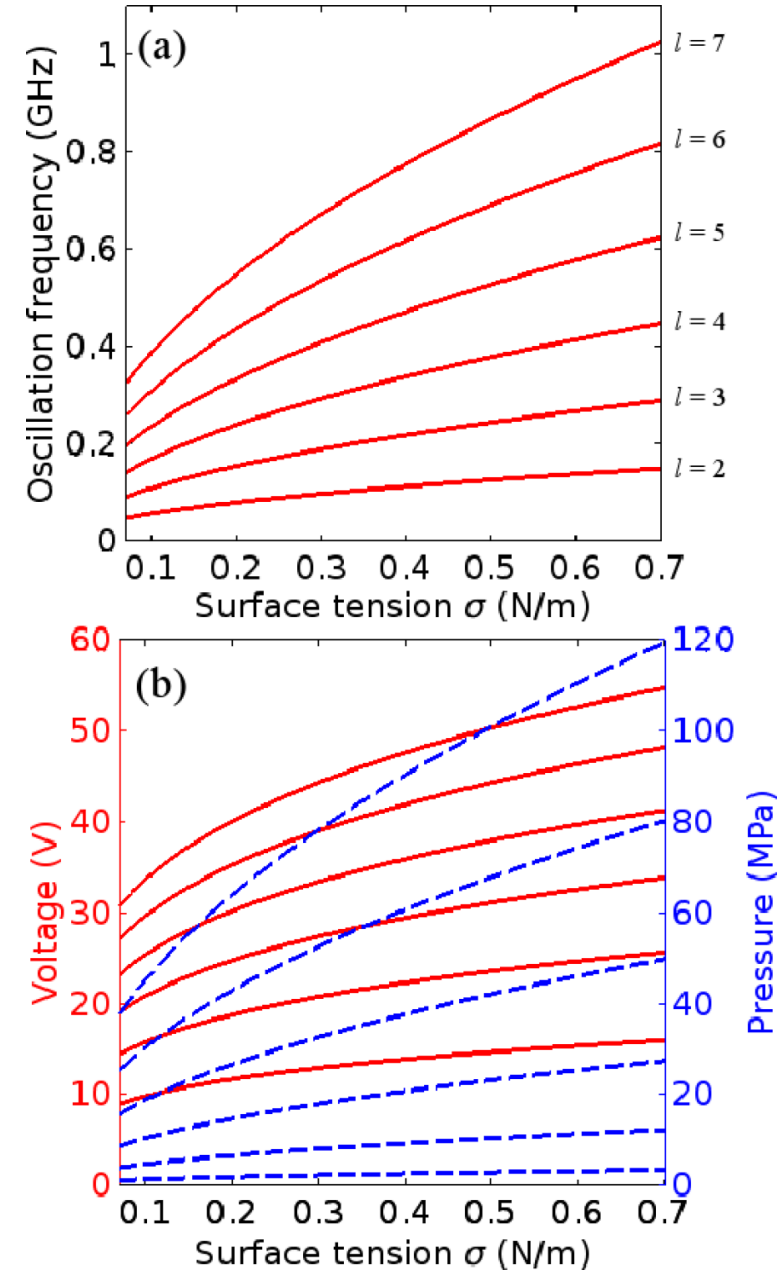

FIG. 3. (a) Resonance oscillation frequency of the modes $l=$ $2, \ldots, 7$ as a function of the surface tension $\sigma$. (b) Voltage (left $y$ axis, solid curves) and pressure (right $y$ axis, dashed curves) required to achieve the peak amplitudes $A_{l} / R=0.3$ for the modes $l=2, \ldots, 7$ as a function of $\sigma$. Similar to panel (a), the lowest (highest) curves correspond to the mode $l=2(l=7)$.

(left $y$ axis, solid curves) and pressure (right $y$ axis, dashed curves) required to achieve the peak amplitudes $A_{l} / R=0.3$ for the modes $l=2, \ldots, 7$, as a function of $\sigma$. We observe that the lowest possible oscillation frequency is $\sim 40 \mathrm{MHz}$ for the mode $l=2$, but for the mode $l=7$ it can reach $1 \mathrm{GHz}$. The applied voltage (pressure) required to achieve $A_{l} / R=0.3$ at these frequencies ranges from $\sim 10(\sim 4 \mathrm{MPa})$ to $\sim 55 \mathrm{~V}(120$ $\mathrm{MPa})$, which are achievable in experiment values [15,23].

Figure 4 shows the calculated scattered light intensity spectra of the nanodroplet at $T=0, T=1$, and $T=1 / 2$ (spherical shape) for the modes $l=2, \ldots, 7$. Three-dimensional (3D), static shapes of the nanodroplet from Fig. 1 were used in the calculations. The the insets in Fig. 4 show the cross-sections of the $3 \mathrm{D}$ shapes at $T=0, T=1$, and $T=1 / 2$ calculated using Eq. (2).

The scattered light intensity spectra were calculated using a customised [43] 3D optical total-field scattered-field finitedifference time-domain (FDTD) method [44] with the spatial discretization $2 \mathrm{~nm}$. We use the dielectric permittivity function of liquid gallium [19], which is a good model for gallium-
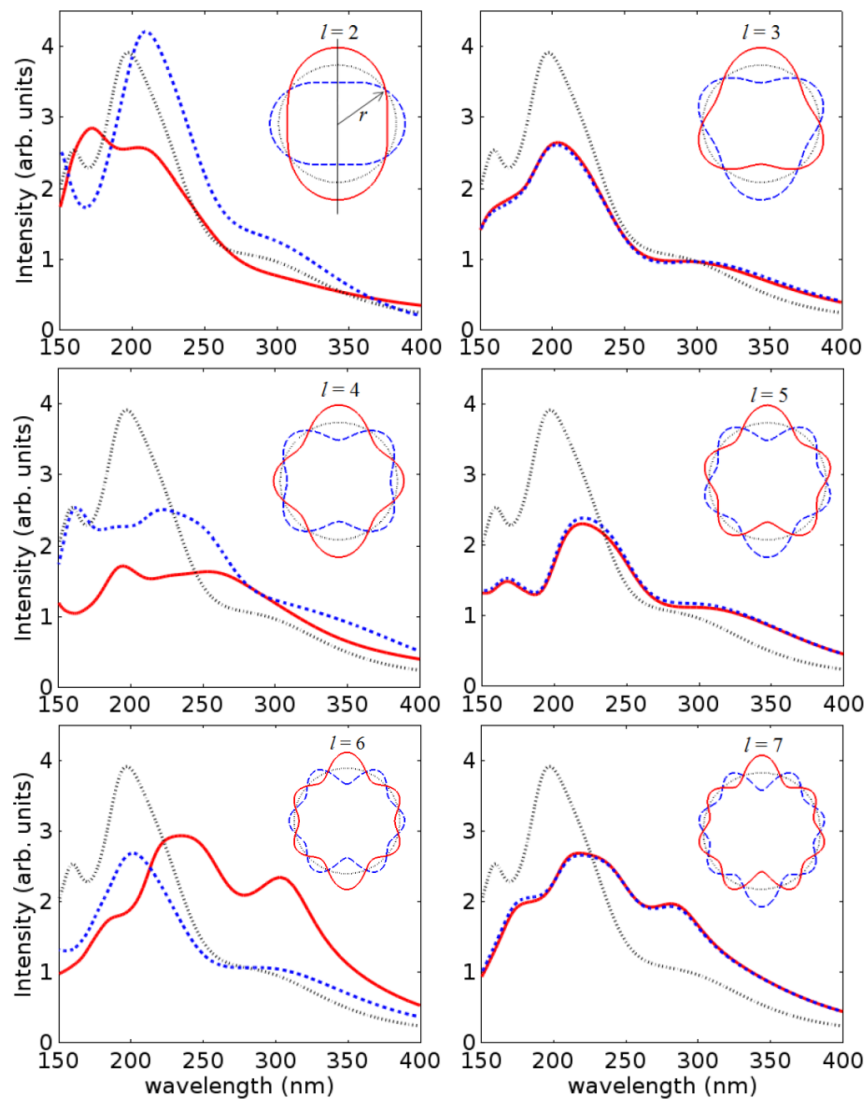

FIG. 4. Scattered light intensity spectra calculated for the static three-dimensional (3D) shapes assumed by the nanodroplet at $T=0$ (dashed curves), $T=1$ (solid curves), and $T=1 / 2$ (dotted curve) shown in Fig. 1. The insets show the cross-sections of these 3D shapes. The same curve styles are used in the insets and the main panels.

based alloys [42]. In calculations, we ignore the presence of a nanometers-thin native gallium oxide layer formed on top of the liquid-gallium alloy because this layer is easily removable [20].

The optical excitation source is a plane wave normally incident onto the nanodroplet. Light transmitted through the computational domain with a single nanodroplet is detected by a probe, and its intensity is normalized to the intensity of the incident light such that the values $>1$ indicate a field enhancement due to the plasmon resonance. The employed computational scheme simulates an experimentally important scenario of light scattering measurements from a liquid sample [37] such as an aqueous solution of liquid-metal nanodroplets [17]. Very often, in those measurement only light scattered in the forward direction is detected. We note that the calculated spectra of the transmitted light may vary when the angle between the direction of the incident light and the probe is varied, which is also possible in experiment, and in that case spectral variations are especially expected when asymmetric capillary oscillations of the nanodroplet are excited (see below).

In Fig. 4, we observe changes in the optical spectrum in the 150-300-nm range due to the shape deformation of the nanodroplet. In particular, as compared to the spherical shape 
$(T=1 / 2)$, the plasmon resonance peaks shift in frequency and their intensity is changed. Moreover, additional resonance peaks appear in the spectra for the higher-order oscillation modes.

For the modes $l=2,4,6$ the spectra produced by the modal shapes at $T=0$ and $T=1$ are different, but for the modes $l=3,5,7$ they coincide to almost graphical accuracy. Hence, the incident light senses more variations in the nanodroplet's shape tuned on one of its even modes than when an odd mode is excited. This result is attributed to the symmetry (asymmetry) of the even (odd) modes with respect to the horizontal axis in the insets in Fig. 4. Whereas the deformed nanodroplet shapes for, e.g., the $l=3$ mode are the same but rotated by $\pi$ radians, for the mode $l=4$ they are different. This results in different optical properties.

\section{CONCLUSION}

We propose and theoretically analyze a room-temperature liquid-metal nanodroplet as a dynamically reconfigurable plasmonic structure, which may be used as an optical nanoantenna having applications in nanoscale optical filters, sensors, optomechanic systems, and transducers of electrical and mechanical forces into optical signals (e.g., nanoscale microphones). The ability to manipulate the plasmon resonances originates from capillary oscillations of the nanodroplets, which may have the peak amplitudes of $\sim 30 \%$ of the undeformed radius at attainable in experiments levels of the oscillation driving force such as applied ac voltage of $\sim 10-50 \mathrm{~V}$ or pressure of $\sim 5-100$ $\mathrm{MPa}$. It is noteworthy that the predicted large changes in the shape of the liquid-metal nanodroplets cannot be achieved in solid-metal nanoparticles [45] due to mechanical hardness of the latter. Although oscillations with comparable amplitudes are possible with gas nanobubbles in liquids [24], nanobubbles have a low refractive index contrast with the host liquid ( $\Delta n \approx 0.33$ in water) and thus require auxiliary plasmonic nanostructures to enable their interaction with the incident light [24]. In contrast, liquid-metal nanodroplets combine both acoustic and optical properties.

Furthermore, the ability to manipulate the surface tension of liquid-gallium metal alloys [20] opens up opportunities to tune the oscillation frequency from several $\mathrm{MHz}$ to several $\mathrm{GHz}$. This is difficult to achieve in solid-metal nanoparticles that vibrate mostly at frequencies $>1 \mathrm{GHz}$ and these frequencies cannot be tuned. Finally, liquid-gallium alloy nanodroplets may be frozen at temperatures $<10-15^{\circ} \mathrm{C}$ or so [16], which converts them into solid-state nanoparticles with virtually the same plasmonic properties [19] but having one of the complex 3D shapes shown in Fig. 1. Thus, variations of the temperature around the freezing point of the liquid-gallium alloy may be used to switch the nanodroplet from the liquid to solid phase and vice versa. However, because liquid-gallium alloys tend to supercool below the freezing point, the liquid nanodroplet has to be perturbed to create crystal nucleation sites required to initiate freezing.

Finally, we note that liquid-metal nanodroplets may be employed inside dual-purpose liquid-core optical fibers [23]. Such optical fibres are designed such that they may guide light inside their water-filled cores (refractive index $n=1.33$ ) formed inside Teflon tubings with $n=1.29$. Significantly, Teflon tubings also enable acoustic impedance matching between water and an optical fiber. The possibility to achieve impedance matching was also demonstrated in [38]. However, tubings with $n>1.33$ were employed in [38], making them not appropriate for waveguiding of light. In our case because of the acoustic impedance matching, when a section of the fiber is immersed into the water tank in Fig. 2(a) instead of the cuvette, ultrasound emitted by the transducer propagates through the fiber without scattering [23], thereby exciting capillary oscillations of nanodroplets more efficiently than when a cuvette is employed. The ends of the water-core fiber are not immersed in water. One end is used to couple light to the fiber core and the other end is used to collect the output light. This simplifies the excitation of plasmon modes of nanodroplets located inside the water-filled fiber core because light is localized mostly in those regions where nanodroplets are located. Consequently, many technical challenges due to the need to focus light on nanodroplets located inside the water tank [38] no longer apply.

\section{ACKNOWLEDGMENTS}

This work was supported by the Australian Research Council (ARC) through its Centre of Excellence for Nanoscale BioPhotonics (CE140100003), LIEF program (LE160100051), and Future Fellowship (FT1600357). This research was undertaken on the NCI National Facility in Canberra, Australia, which is supported by the Australian Commonwealth Government.
[1] V. Klimov, Nanoplasmonics (Pan Stanford, Singapore, 2014).

[2] L. Novotny and N. van Hulst, Nat. Photon. 5, 83 (2011).

[3] M. Agio and A. Alù, Optical Antennas (Cambridge University Press, Cambridge, England, 2013).

[4] I. S. Maksymov, I. Staude, A. E. Miroshnichenko, and Y. S. Kivshar, Nanophotonics 1, 65 (2012).

[5] I. S. Maksymov, Rev. Phys. 1, 36 (2016).

[6] A. Byrnes, R. Pant, E. Li, D.-Y. Choi, C. G. Poulton, S. Fan, S. Madden, B. Luther-Davies, and B. J. Eggleton, Opt. Express 20, 18836 (2012).

[7] J. P. Turpin, J. A. Bossard, K. L. Morgan, D. H. Werner, and P. L. Werner, Int. J. Antennas Propagat. 2014, 429837 (2014).
[8] M. Lapine, D. Powell, M. Gorkunov, I. Shadrivov, R. Marqués, and Yu. S. Kivshar, Appl. Phys. Lett. 95, 084105 (2009).

[9] J. T. Bernhard, Synthesis Lectures on Antennas: Reconfigurable Antennas (Morgan \& Claypool, Williston, VT, 2007).

[10] C. Brüggemann, A. V. Akimov, B. A. Glavin, V. I. Belotelov, I. A. Akimov, J. Jäger, S. Kasture, A. V. Gopal, A. S. Vengurlekar, D. R. Yakovlev, A. J. Kent, and M. Bayer, Phys. Rev. B 86, 121401(R) (2012).

[11] M. Beebe, L. Wang, S. E. Madaras, J. M. Klopf, Z. Li, D. Brantley, M. Heimburger, R. A. Wincheski, S. Kittiwatanakul, J. Lu, S. A. Wolf, and R. A. Lukaszew, Opt. Express 23, 13222 (2015). 
[12] A. F. Coskun, A. E. Cetin, B. C. Galarreta, D. A. Alvarez, H. Altug, and A. Ozcan, Sci. Rep. 4, 6789 (2014).

[13] C. D. Geddes, Reviews in Plasmonics 2016 (Springer, Berlin, 2017).

[14] Rayleigh, Proc. R. Soc. Lond. 29, 71 (1879).

[15] J. M. Oh, S. H. Ko, and K. H. Kang, Langmuir 24, 8379 (2008).

[16] N. B. Morley, J. Burris, L. C. Cadwallader, and M. D. Nornberg, Rev. Sci. Instrum. 79, 056107 (2008).

[17] Y. Lin, Y. Liu, J. Genzer, and M. D. Dickey, Chem. Sci. 8, 3832 (2017).

[18] M. Yarema, M. Wörle, M. D. Rossell, R. Erni, R. Caputo, L. Protesescu, K. V. Kravchyk, D. N. Dirin, K. Lienau, F. von Rohr, A. Schilling, M. Nachtegaal, and M. V. Kovalenko, J. Am. Chem. Soc. 136, 12422 (2014).

[19] M. W. Knight, T. Coenen, Y. Yang, B. J. M. Brenny, M. Losurdo, A. S. Brown, H. O. Everitt, and A. Polman, ACS Nano 9, 2049 (2015).

[20] M. R. Khan, C. B. Eaker, E. F. Bowden, and M. D. Dickey, PNAS 111, 14047 (2014).

[21] I. S. Maksymov and A. D. Greentree, Sci. Rep. 6, 32892 (2016).

[22] Y. Ma, Q. Huang, T. Li, J. Villanueva, N. H. Nguyen, J. Friend, and D. J. Sirbuly, ACS Photonics 3, 1762 (2016).

[23] I. S. Maksymov and A. D. Greentree, Opt. Express 25, 7496 (2017).

[24] I. S. Maksymov and A. D. Greentree, Phys. Rev. A 95, 033811 (2017).

[25] P. V. Zinin and J. S. Allen, III, Phys. Rev. E 79, 021910 (2009).

[26] Y. Lu, Q. Hu, Y. Lin, D. B. Pacardo, C. Wang, W. Sun, F. S. Ligler, M. D. Dickey, and Z. Gu, Nat. Commun. 6, 10066 (2015).

[27] Th. Crouzil and M. Perrin, J. Europ. Opt. Soc. Rap. Public. 8, 13079 (2013).

[28] R. Dahan, L. L. Martin, and T. Carmon, Optica 3, 175 (2016).

[29] S. Maayani, L. L. Martin, S. Kaminski, and T. Carmon, Optica 3, 552 (2016).
[30] S. Chandrasekhar, Hydrodynamic and Hydromagnetic Stability (Oxford University Press, Oxford, 1961).

[31] C. Miller and L. Scriven, J. Fluid Mech. 32, 417 (1968).

[32] J. A. Tsamopoulos and R. A. Brown, J. Fluid Mech. 127, 519 (1983).

[33] T. W. Patzek, R. E. Benner, Jr., O. Basaran, and L. E. Scriven, J. Comp. Phys. 97, 489 (1991).

[34] O. Basaran, J. Fluid Mech. 241, 169 (1992).

[35] G. B. Foote, J. Comp. Phys. 11, 507 (1973).

[36] C. T. Alonso, JPL Proc. Intern. Colloq. Drops Bubbles 1, 139 (1974).

[37] W. Deng, E. M. Goldys, M. M. J. Farnham, and P. M. Pilowsky, Am. J. Physiol. Regul. Integr. Comp. Physiol. 307, R1292 (2014).

[38] O. Simandoux, A. Prost, J. Gateau, and E. Bossy, Photoacoustics 3, 20 (2015).

[39] C. Raufaste, G. R. Chagas, T. Darmanin, C. Claudet, F. Guittard, and F. Celestini, Phys. Rev. Lett. 119, 108001 (2017).

[40] Z. Wan, H. Zeng, and A. Feinerman, ASME. J. Fluids Eng. 129, 388 (2007).

[41] A. V. Diebold, A. M. Watson, S. Holcomb, C. Tabor, D. Mast, M. D. Dickey, and J. Heikenfeld, J. Micromech. Microeng. 27, 025010 (2017).

[42] D. Morales, N. A. Stoute, Z. Yu, D. E. Aspnes, and M. D. Dickey, Appl. Phys. Lett. 109, 091905 (2016).

[43] I. S. Maksymov, A. A. Sukhorukov, A. V. Lavrinenko, and Y. S. Kivshar, IEEE Antennas Wireless Propagat. Lett. 10, 143 (2011).

[44] A. Taflove and S. C. Hagness, Computational Electrodynamics: The Finite-Difference Time-Domain Method (Artech House, Boston, 2005).

[45] P. V. Ruijgrok, P. Zijlstra, A. L. Tchebotareva, and M. Orrit, Nano Lett. 12, 1063 (2012). 